\title{
Pump thrombosis: Do we have the answer to the riddle?
}

\author{
Francis D. Pagani, MD, PhD
}

\author{
From the Department of Cardiac Surgery, University of Michigan, Ann Arbor, Mich. \\ Disclosures: Author has nothing to disclose with regard to commercial support. \\ Received for publication Feb 28, 2017; accepted for publication March 2, 2017; available ahead of print March 24, \\ 2017. \\ Address for reprints: Francis D. Pagani, MD, PhD, Department of Cardiac Surgery, 5161 Cardiovascular Center, \\ SPC 5864, 1500 East Medical Center Drive, Ann Arbor, MI 48109 (E-mail: fpagani@umich.edu). \\ J Thorac Cardiovasc Surg 2017;153:1509-10 \\ $0022-5223 / \$ 36.00$ \\ Copyright (C) 2017 by The American Association for Thoracic Surgery \\ http://dx.doi.org/10.1016/j.jtcvs.2017.03.007
}

Despite significant improvements in design and patient outcomes with implantable ventricular assist devices (VADs), the cumulative adverse event burden of this therapy poses significant challenges to the field. ${ }^{1-3}$ Of the adverse events, those related to the hematologic system, that is, pump thrombosis, stroke, and mucosal bleeding, have been particularly troublesome and have received significant attention over the past few years. ${ }^{1-3}$ In this issue of the Journal, Dr Massicotte and colleagues ${ }^{4}$ have submitted a thoughtful review on the issue of thrombogenicity and pump thrombosis. These authors have stressed the multifactorial nature of the problem that has increased the difficulty in seeking a single solution to the issue. The major factors associated with pump thrombosis as highlighted by Dr Massicotte and colleagues include (1) pump design; (2) patient factors, such as demographics, inherited coagulable disorders, or compliance-related issues; and (3) management issues, such as implant technique and variation in physician treatments. The fact that significant variation exists in rates of pump thrombosis across clinical sites and countries supports the multifactorial nature of the problem. Dr Massicotte and colleagues also raise the challenges of pump thrombosis in the pediatric field and the complexity of issues associated with mechanical circulatory support in children, including the adoption of pumps designed for use in adults being used in the pediatric population and the complex and varying anatomy and biology of children with congenital heart disease. One major theme stressed in the review is the issue of VAD design and thrombogenicity. Is there hope for the future? Recent published data from the MOMENTUM 3 clinical trial perhaps sheds some light on the topic on pump thrombosis and stresses the importance of technology as a critical factor in solving this issue. ${ }^{5}$ The MOMENTUM 3 trial was a prospective, multicenter, randomized clinical trial comparing the HeartMate 3 (Thoratec Corp, Pleasanton, Calif) with the HeartMate II pump for patients with advanced stage heart failure. Of note, the HeartMate 3 pump demonstrated superiority over the HeartMate II pump with respect to the primary end point. The major reason for superior performance of the HeartMate 3 over that of the HeartMate

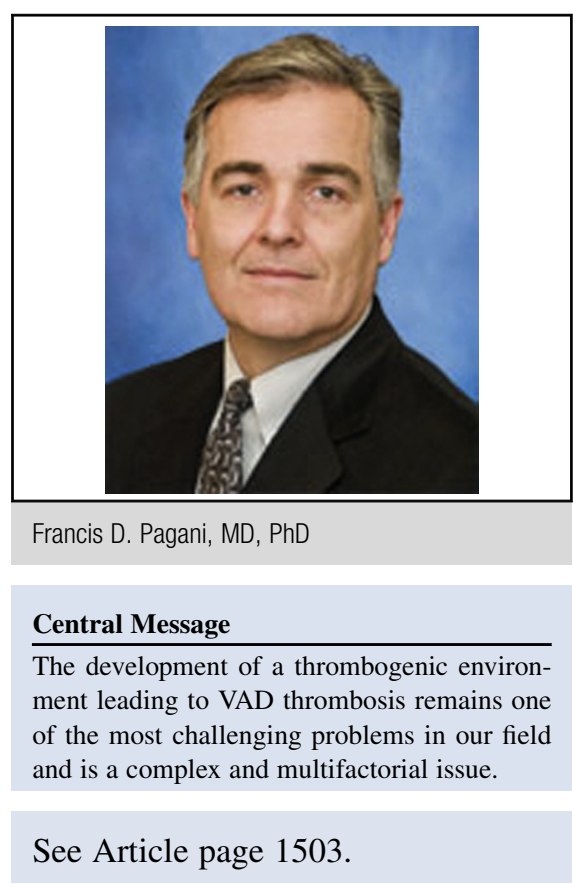

II was the significantly lower incidence of need for pump exchange ( 1 event [0.7\%] vs 11 events [7.7\%], respectively) as a result of complete absence of pump thrombosis in the HeartMate 3, whereas the HeartMate II experienced a $10.1 \%$ incidence of confirmed or suspected pump thrombosis. What do these data tell us? These data suggest that innovative VAD designs can solve an important biological issue. Although both pumps were similarly and randomly exposed to variations in patient management and patientrelated issues, the HeartMate 3 was more resilient to pump thrombosis. This observation does not undermine the importance of patient management and patient factors contributing to pump thrombosis for those pumps with designs less resilient to pump thrombosis. What is important is that we now know that technology can succeed in resolving an important adverse event. Even more important is can we apply this to the pediatric population? As stated by Dr Massicotte and colleagues, greater collaboration and support with industry are needed to assist in the development of novel pump systems, particularly for the challenging area of pediatric mechanical circulatory support.

\section{References}

1. Kirklin JK, Naftel DC, Pagani FD, Kormos RL, Myers S, Acker MA, et al. Pump thrombosis in the Thoratec HeartMate II device: an update analysis of the INTERMACS Registry. J Heart Lung Transplant. 2015;34:1515-26.

2. Najjar SS, Slaughter MS, Pagani FD, Starling RC, McGee EC, Eckman P, et al. An analysis of pump thrombus events in patients in the HeartWare ADVANCE bridge 
to transplant and continued access protocol trial. J Heart Lung Transplant. 2014; 33:23-34.

3. Starling RC, Moazami N, Silvestry SC, Ewald G, Rogers JG, Milano CA, et al. Unexpected abrupt increase in left ventricular assist device thrombosis. $N$ Engl J Med. 2014;370:33-40.
4. Massicotte P, Snyder T, Stulak J, Kreuziger LB. VAD thrombosis: mind your P's and Q's: pumps, patients and pills. J Thoracic Cardiovasc Surg. 2017;153:1503-6.

5. Mehra MR, Naka Y, Uriel N, Goldstein DJ, Cleveland JC Jr, Colombo PC, et al. A fully magnetically levitated circulatory pump for advanced heart failure. $N$ Engl J Med. 2017;376:440-50. 